\title{
Nonlinear dynamics of polariton scattering in semiconductor microcavity: bistability vs stimulated scattering
}

\author{
N. A. Gippius, ${ }^{a}$ S. G. Tikhodeev,${ }^{a}$ V. D. Kulakovskii, ${ }^{b}$ D. N. Krizhanovskii, ${ }^{b}$ and A. I. Tartakovskii ${ }^{b}$ \\ (a) General Physics Institute, Russian Academy of Sciences, Vavilova 38, Moscow 119991, Russia \\ (b) Institute of Solid State Physics, RAS, Chernogolovka, 142432 Russia
}

\begin{abstract}
We demonstrate experimentally an unusual behavior of the parametric polariton scattering in semiconductor microcavity under a strong cw resonant excitation. The maximum of the scattered signal above the threshold of stimulated parametric scattering does not shift along the microcavity lower polariton branch with the change of pump detuning or angle of incidence but is stuck around the normal direction. We show theoretically that such a behavior can be modelled numerically by a system of Maxwell and nonlinear Schrödinger equations for cavity polaritons and explained via the competition between the bistability of a driven nonlinear MC polariton and the instabilities of parametric polariton-polariton scattering.
\end{abstract}

PACS numbers: $71.36 .+\mathrm{c}, 42.50 . \mathrm{p}, 42.65 . \mathrm{k}, 42.65 . \mathrm{Sf}, 68.65 .+\mathrm{g}$

The effect of parametric scattering of semiconductor microcavity (MC) polaritons has been established recently 1, 2, 3, 4, 5, 6, 7, 8, 9, 10, 11, 12 and attracted a significant interest. Extremely large parametric gains [1, 10] and striking transformations of the polariton parametric luminescence with the increase of intensity of resonant coherent excitation 2, 4, 5, [6, 9] have been demonstrated and analyzed theoretically [3, 7, 8, 9, 11]. However, only the case of specific pump conditions known as magic angle excitation, when the parametric scattering threshold intensity is minimum, has been investigated so far in detail in case of stationary excitation.

In this Letter we investigate the parametric MC polariton-polariton scattering varying the cw pump angle and energy away from the magic angle conditions. According to the existent theory [7, 8, 9], the maxima of scattered signal have to shift along the blueshifted and renormalized lower polariton (LP) branch. To the contrary, we found experimentally that above the threshold of stimulated parametric scattering the signal maximum stays approximately in the normal direction, notwithstanding the change of the excitation conditions [13].

We show numerically that a qualitatively similar behaviour can be demonstrated by a system of Maxwell and nonlinear Schrödinger equations for MC quantum well excitons, accounting for the exciton-cavity photon coupling into MC polariton and polariton-polariton scattering. In the real life, many other scattering processes can play a role, e.g., polariton-phonon scattering 14], polariton-free carrier scattering [15]. But it turns out that the polariton-polariton scattering alone demonstrates already sharp transformation of the scattering process, which results in the jump of the scattered signal from the oblique to normal direction, in a qualitative agreement with the experiment.

In the part of polariton-polariton scattering, our description is analogous to that in [7, 8, [9]. As in [8], we neglect the saturation term, responsible for the Pauli

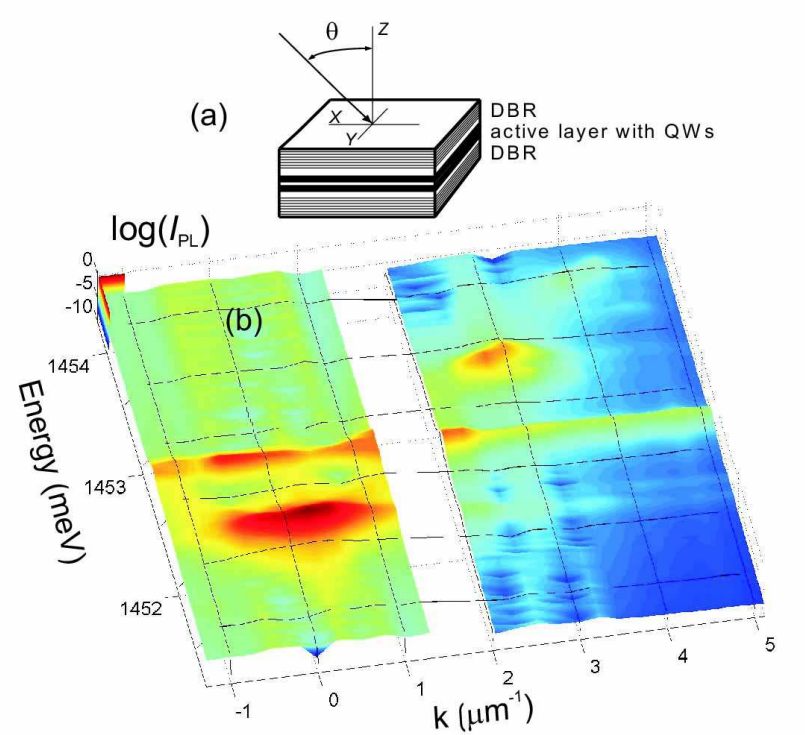

FIG. 1: The experimental geometry $(a)$; measured energymomentum spectra of the LP photoluminescence under a resonant excitation at $\hbar \Omega_{0}=1452.7 \mathrm{meV}$ and $\vartheta=12.5^{\circ}$ with intensity above the scattering threshold $(b)$.

principle in exciton-photon interaction. It can be shown that its influence is not strong in case of MC with our parameters. However, one difference is important. We treat the amplitude of the driven polariton mode not as a fixed external parameter, but as an internal parameter established in the nonlinear system under a fixed external pump. Well known in the theory of driven nonlinear optical cavities without a polariton resonance (see, e.g., in 16, 17]), this description allows to explain bistability, symmetry breaking, pattern formation, bifurcations to chaos. As we show in this Letter, the bistability can play an important role in the nonlinear kinetics of $\mathrm{MC}$ polariton scattering as well.

Experimentally, we have investigated photoluminescence (PL) from GaAs/AlAs MC containing six 10-nmthick InGaAs quantum wells (QW's) in $3 / 2 \lambda$ GaAs cavity 


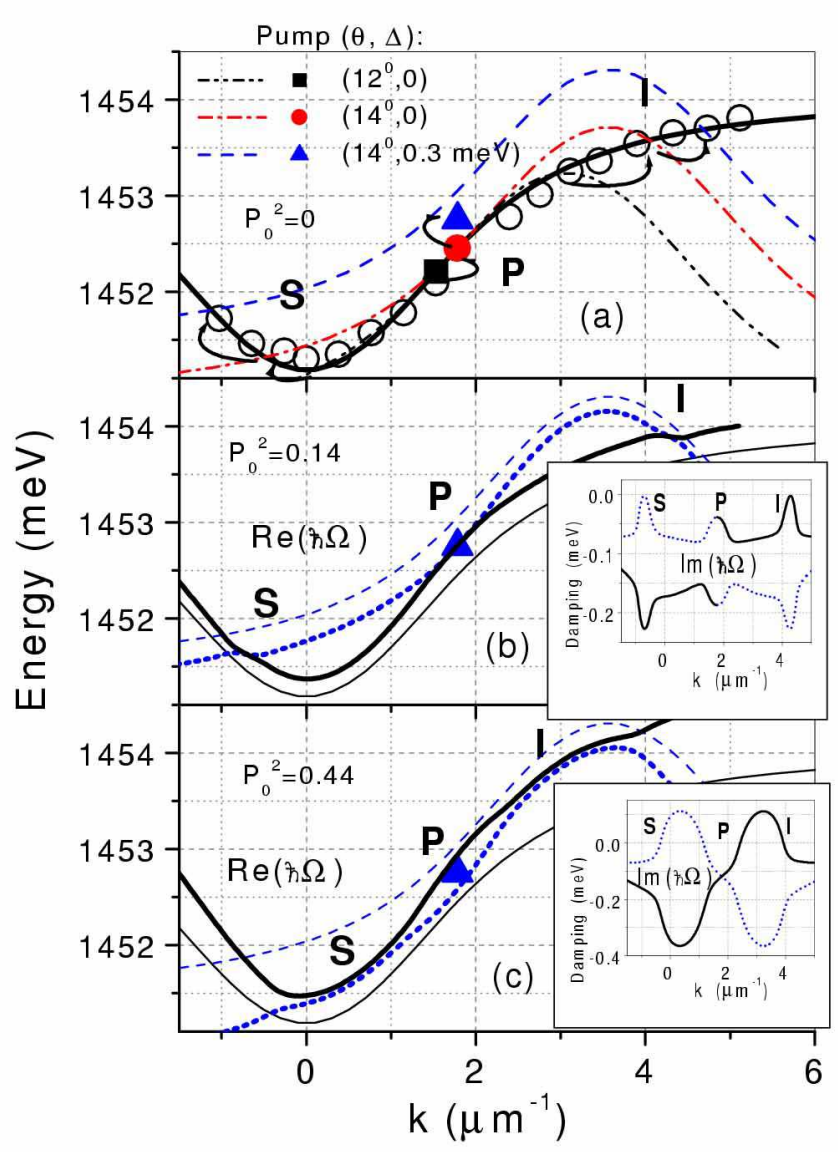

FIG. 2: Lower polariton branches: measured (circles) and calculated for $\left|\mathcal{P}_{0}\right|^{2}=0(a), 0.14(b)$, and $0.44(c)$. Inserts in panels $b, c$ show the corresponding dampings. The lines are: signal (solid) and idler (dashed). Dash-dot-dot, dash-dot and dash lines in panel $a$ are idlers calculated for $\left|\mathcal{P}_{0}\right|^{2}=0$ and different pump angles and detunings $(\vartheta, \Delta)=\left(12^{\circ}, 0\right)$ - magic excitation, $\left(14^{\circ}, 0\right)$ and $\left(14^{\circ}, 0.3 \mathrm{meV}\right) .\left[\Delta=\hbar \Omega_{0}-E_{\mathrm{LP}}\left(\mathbf{k}_{0}\right)\right.$. Square, circle, and triangle are the corresponding pump $(\mathrm{P})$ locations. Thin lines in panels $b, c$ are signal and idler at $\left|\mathcal{P}_{0}\right|^{2}=0$ from panel $a$.

with cavity mode (cavity mode - QW exciton detuning $-0.5 \mathrm{meV}$ ), see the experimental geometry in Fig. 1 $a$. A sample was mounted in a cryostat with a wide angular access $\left(70^{\circ}\right)$ at a controlled temperature of $\mathrm{T}=1.8-20 \mathrm{~K}$. The experiment was done under excitation with a tunable cw Ti-Sapphire laser under a changeable angle of incidence $\vartheta_{0}$. The angle resolved measurements of the $\mathrm{MC}$ emission and the use of $\mathrm{cw}$ excitation have allowed to fix both the energy and wavevector of the emission with a high precision. The details on the sample and experimental set-up can be found in [4].

Figure $1 \mathrm{~b}$ shows the distribution of the scattered light vs. energy $\hbar \omega$ and in-plane momentum $k=$ $\hbar \omega \sin \vartheta / c$. The excitation is at $\vartheta_{0}=12.5^{\circ}$ and $\hbar \Omega_{0}=$ $1452.7 \mathrm{meV}\left(k_{0} \approx 1.6 \mu \mathrm{m}^{-1}\right)$, with high excitation power of $880 \mathrm{~W} / \mathrm{cm}^{2}$, which is above the threshold of stimulated parametric scattering. Figure shows two pronounced emission maxima at $k \gtrsim 0$ and $k \lesssim 2 k_{0}$. A closer look shows that this cannot be explained within the existent theory of the MC parametric scattering [7, 8, 9], even if the LP spectra renormalization is taken into account.
The bare LP dispersion $E_{\mathrm{LP}}(\mathbf{k})$ has been determined experimentally from the angle resolved PL measurements under a weak above band gap excitation, see in Fig. $21 a$. The inflexion point of the LP branch is at $\approx 12^{\circ}$, which corresponds to momentum $\approx 1.5 \cdot 10^{4} \mathrm{~cm}^{-1}$ and energy $1452.2 \mathrm{meV}$.

At low excitation intensity the LP polariton-polariton parametric scattering should take place with the energy and in-plane momentum conservation, $\mathbf{k}+\mathbf{k}^{\prime}=$ $2 \mathbf{k}_{0}, \quad E_{\mathrm{LP}}(\mathbf{k})+E_{\mathrm{LP}}\left(\mathbf{k}^{\prime}\right)=2 \hbar \Omega_{0}$. Two sharp emission peaks, signal (S) at $\mathbf{k}_{s}$ and idler (I) at $\mathbf{k}_{i}$, are predicted at the intersection of the signal and idler branches $E_{\mathrm{LP}}(\mathbf{k})$ and $2 \hbar \Omega_{0}-E_{\mathrm{LP}}\left(2 \mathbf{k}_{0}-\mathbf{k}\right)$, see in Fig $2 a$. Under a weak pump into the inflexion point (magic angle) the signal peak is predicted at $k_{s} \approx 0$. With the shift of the pump angle and energy away from the inflection point $\mathrm{S}$ and I peaks shift along the LP branch towards $k_{s}<0, k_{i}>2 k_{0}$ as shown by arrows in Fig. $2 a$.

With increasing the pump intensity the signal and idler branches become renormalised via the parametric coupling [3, 7], see in Fig. 20b. Simultaneosly the dampings of signal and idler branches at the peak positions approach zero (see insert in panel $b$ ). At some critical pump intensity the damping changes sign (becomes gain), which signals the instability and means the threshold of the parametric stimulated scattering. However, the prediction within this theoretical model is still $k_{s}<0, k_{i}>2 k_{0}$, whereas on the experiment we observe the signal and idler maxima at $k_{s} \gtrsim 0, k_{i} \lesssim 2 k_{0}$.

In order to model the polariton scattering dynamics, we solve numerically the system of coupled equations for $\mathcal{E}_{\mathrm{QW}}$, the electric field on the $\mathrm{QW}$, and $\mathcal{P}(k, t)$, the exciton polarisation integrated over the $\mathrm{QW}$ thickness,

$$
\begin{aligned}
& {\left[i \hbar \frac{d}{d t}-E_{\mathrm{MC}}\right] \mathcal{E}_{\mathrm{QW}}(k, t)=\alpha \mathcal{E}_{\mathrm{ext}}(k, t)+\beta \mathcal{P}(k, t),} \\
& {\left[i \hbar \frac{d}{d t}-E_{X}\right] \mathcal{P}(k, t)=A \mathcal{E}_{\mathrm{QW}}(k, t)+\xi(t)} \\
& \quad+F \sum_{q, q^{\prime}} \mathcal{P}(q, t) \mathcal{P}\left(q^{\prime}, t\right) \mathcal{P}^{*}\left(q+q^{\prime}-k, t\right) .
\end{aligned}
$$

Here $\mathcal{E}_{\text {ext }}=\mathcal{E}_{\text {ext }}(t) e^{-i \Omega_{0} t} \delta_{k, k_{0}}$ is the external electric field of the incoming pump far from the MC which is treated as coherent macro-occupied mode with fixed $\hbar \Omega_{0}$ and $k_{0} . \mathcal{E}_{\text {ext }}(t)$ is the pump amplitude, changing slowly with time. $E_{\mathrm{MC}}(k)$ and $E_{X}$ are empty microcavity and QW exciton frequencies. $F$ and $A$ are the QW exciton interaction constant and susceptibility, $\alpha(k)$ and $\beta(k)$ are the $\mathrm{MC}$ response coefficients. In these notations, the $\mathrm{MC}$ polariton Rabi frequency is $\sqrt{A \beta}$. Both $E_{\mathrm{MC}}(k)$ and $E_{X}$ are complex, with imaginary parts accounting for finite lifetimes of the MC mode and QW exciton. $\xi(k, t)$ is the stochastic Langevin force. $E_{\mathrm{MC}}(k), \alpha(k)$, and $\beta(k)$ are calculated via the scattering matrices of the upper and lower MC Bragg mirrors. $E_{X}$ and $A$ are fitted parameters. The energy is measured in $\mathrm{meV}$, the units of polarisation and electric field are such that $F=1$. The 

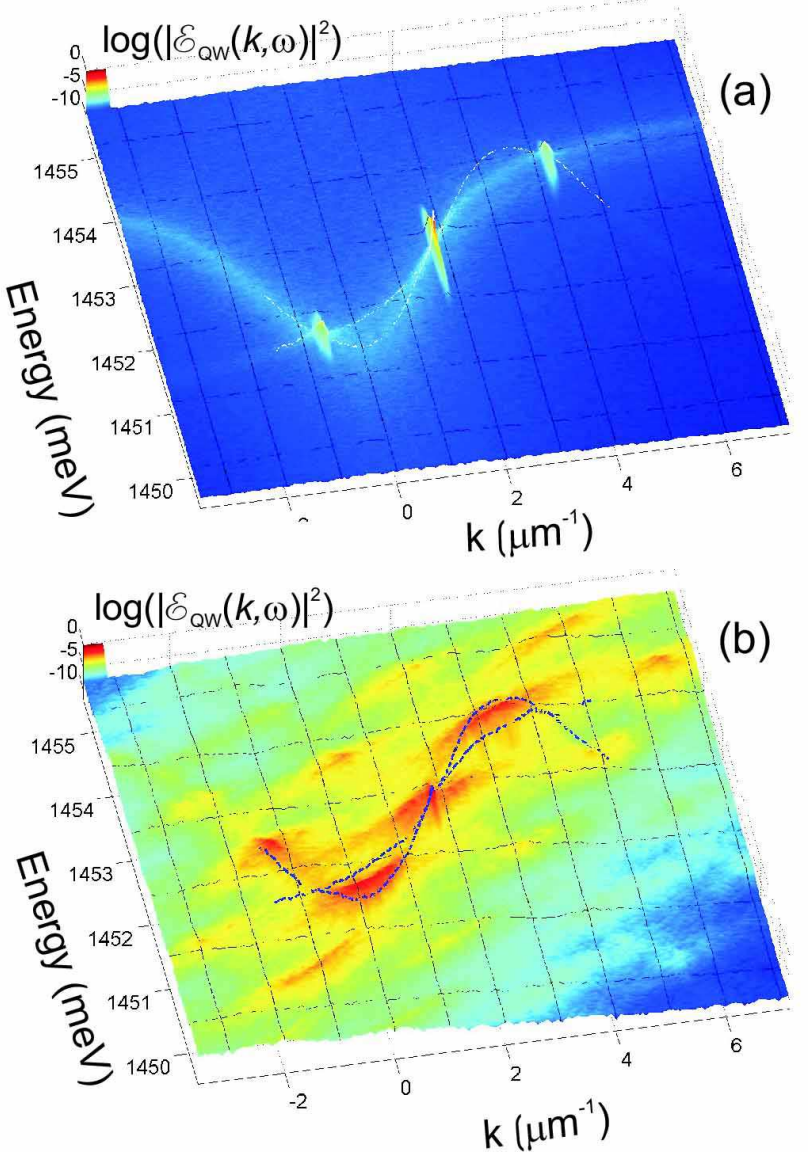

FIG. 3: The calculated time-integrated energy-momentum spectra of the scattered light from MC when the pump pulse amplitude is slightly below $(a)$ and above $(b)$ the threshold. Dotted lines are the signal and idler branches from Fig. $2 b$ calculated at the parametric scattering threshold $\left|\mathcal{P}_{0}\right|^{2}=0.14$.

in-plane momentum is two-dimensional, but we solve numerically only a simplified one-dimensional model.

Equation (11) is the Maxwell equation with the exciton polarization in the resonance approximation for the cavity. Equation (2) is the inhomogeneous nonlinear Schrödinger equation with damping and two types of sources: coherent external pump and stochastic Langevin noise. Eqs.(12) allow to describe the influence of coherent pump and quantum fluctuations simultaneously in a very convenient for numerical modelling way.

The numerical solution of Eqs. (12) for long ( $\approx 1 \mathrm{~ns}$ ) nearly rectangular excitation pulses demonstrates a sharp threshold-like transition from a picture agreeing with the described above stationary model to a completely different one, compare Figs. $3 a$ and $b$. Note that the difference in the pump intensities between panels $a$ and $b$ is $\approx 1 \%$ only. The averaged intensity of scattered noise increases above transition by several orders of magnitude (note the logarithmic vertical scale in Fig. 31).

Figure 4 explains the time kinetics of the scattering above the threshold pump intensity. The shape of the excitation pulse $\mathcal{E}_{\text {ext }}^{2}$ (dash-dotted line in Fig. $4 a$ ) was chosen in such a way that the threshold intensity is ap-
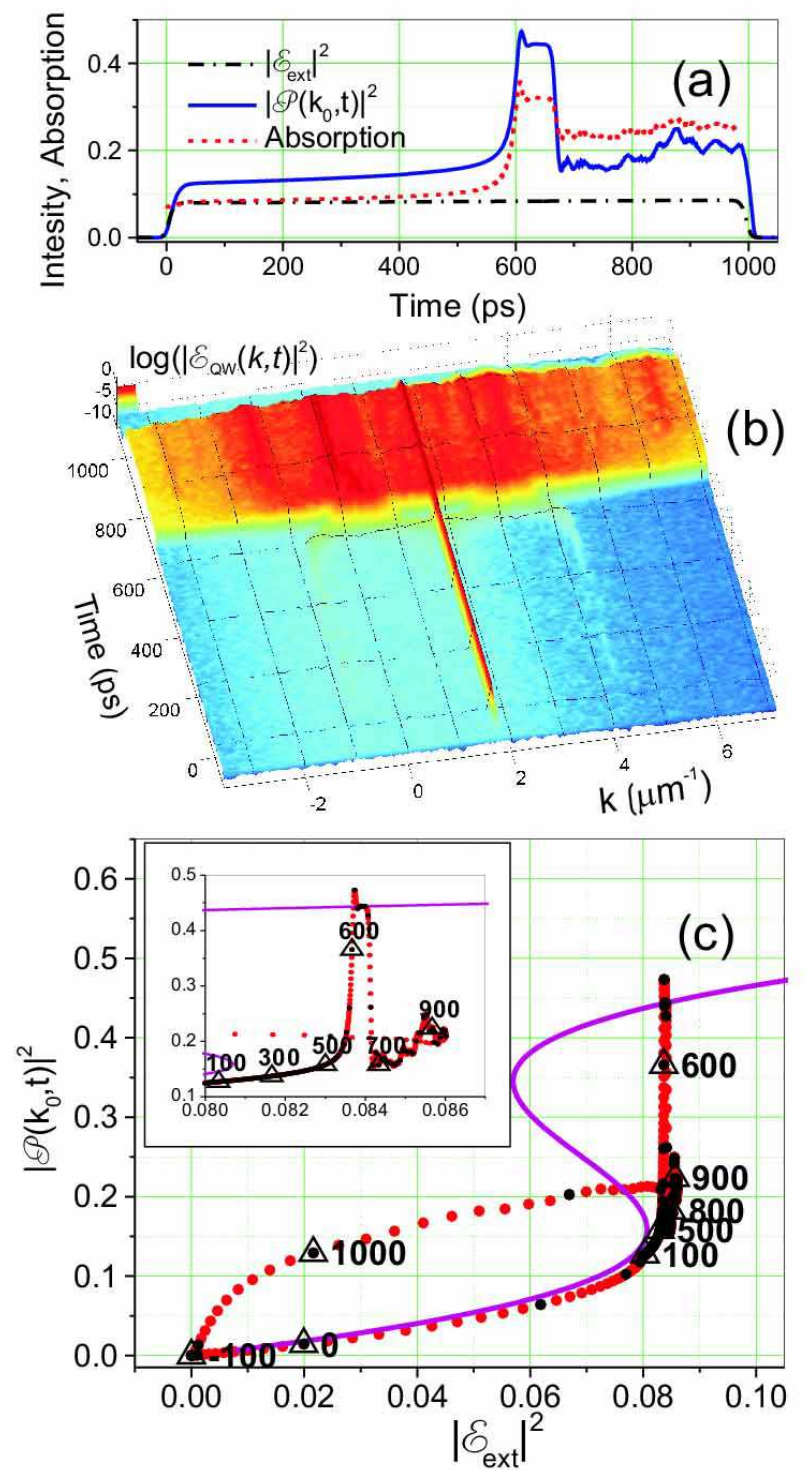

FIG. 4: The time dependences: of input excitation pulse, calculated exciton polarisation and absorption coefficient $(a)$; of angular spectra of the scattered light $(b)$. Exciton polarisation vs pump intensity during the excitation pulse $(c)$. Insert shows the magnified part of the region of transitions. The time delay in panel $c$ between red, black, and triangled dots is 1,10 , and $100 \mathrm{ps}$, respectively; the triangles are labelled by time in ps. Solid S-shaped curve is the solution of Eq. (3).

proached slowly during the pulse duration. Actually two sharp transitions take place (at $t \approx 600$ and $700 \mathrm{ps).}$ Both transitions are characterized by the jumps of the driven mode amplitude $\mathcal{P}_{0}$ itself, see solid line in Fig. 4 4 .

In order to understand these transitions, we have to investigate the stability of solutions of Eqs. (12) in the case of stationary external field $\mathcal{E}_{\text {ext }}(t)=$ const with only one macroscopically filled mode, i.e., of the form $\mathcal{P}(k, t)=\tilde{\mathcal{P}}(k, t)+\delta_{k, k_{0}} \mathcal{P}_{0} e^{-i \Omega_{0} t}$, and $\mathcal{E}_{\mathrm{QW}}(k, t)=$ $\tilde{\mathcal{E}}(k, t)+\delta_{k, k_{0}} \mathcal{E}_{0} e^{-i \Omega_{0} t}$. Here $\left|\tilde{\mathcal{P}} / \mathcal{P}_{0}\right|,\left|\tilde{\mathcal{E}} / \mathcal{E}_{0}\right| \ll 1$. In zero order we get the following cubic equation for the ampli- 
tude of the driven mode $\mathcal{P}_{0}$

$$
\left[\Delta_{0}\left(\Delta_{0}+\Delta_{X C}\right)-A \beta\right] \mathcal{P}_{0}-\Delta_{0} F\left|\mathcal{P}_{0}\right|^{2} \mathcal{P}_{0}=A \alpha \mathcal{E}_{\text {ext }},
$$

where $\Delta_{0}=\hbar \Omega_{0}-E_{\mathrm{MC}}\left(k_{0}\right), \Delta_{X C}=E_{\mathrm{MC}}\left(k_{0}\right)-E_{X}$. Solution of Eq. (3) is shown as the solid S-shaped line in Fig. 4lc. The S-shape is well known in the theory of nonlinear cavities (see, e.g., in Refs. 16, 17]), it brings a bistability into the behaviour of the $\mathrm{MC}$ polaritons.
The influence of the bistability was not analyzed in the existent theories of the MC polariton scattering 3,7 , 8 , 9, 11].

As to the stability conditions, linearising Eqs. (12), we have to solve the linear eigenproblem for parametrically coupled signal $\tilde{\mathcal{E}}, \tilde{\mathcal{P}}(k, t)=\tilde{\mathcal{E}}, \tilde{\mathcal{P}}(k) e^{-i \omega t}$ and idler $\tilde{\mathcal{E}}^{*}, \tilde{\mathcal{P}}^{*}(\bar{k}, t) e^{-2 i \Omega_{0} t}=\overline{\overline{\mathcal{E}}}, \overline{\tilde{\mathcal{P}}}(\bar{k}) e^{-i \omega t}$ (where $\left.\bar{k}=2 k_{0}-k\right)$,

$$
\hbar \omega\left(\begin{array}{c}
\tilde{\mathcal{E}}(k) \\
\tilde{\mathcal{P}}(k) \\
\overline{\tilde{\mathcal{E}}}(\bar{k}) \\
\overline{\tilde{\mathcal{P}}}(\bar{k})
\end{array}\right)=\left(\begin{array}{cccc}
E_{\mathrm{MC}}(k) & \beta & 0 & 0 \\
A & E_{X}+2 F\left|\mathcal{P}_{0}\right|^{2} & 0 & F \mathcal{P}_{0}^{2} \\
0 & 0 & 2 \Omega_{0}-E_{\mathrm{MC}}^{*}(\bar{k}) & -\beta^{*} \\
0 & -\left(F \mathcal{P}_{0}^{2}\right)^{*} & -A^{*} & 2 \Omega_{0}-E_{X}^{*}-2 F^{*}\left|\mathcal{P}_{0}\right|^{2}
\end{array}\right)\left(\begin{array}{c}
\tilde{\mathcal{E}}(k) \\
\tilde{\mathcal{P}}(k) \\
\overline{\tilde{\mathcal{E}}}(\bar{k}) \\
\tilde{\tilde{\mathcal{P}}}(\bar{k})
\end{array}\right)
$$

and check the sign of the imaginary parts of the eigenenergies. Equation (4) appears to be analogous to that discussed in detail in Refs. [7, 8, 9]. We use the MC-X basis instead of the UP-LP one. There is an advantage of the MC-X basis, because the dependence of the interaction constant $F$ on $k$ can be neglected. Then, in the space representation, it corresponds to a local contact interaction $F \int|\mathcal{P}(x)|^{4} d x$, which allows to write a very efficient numerical code for Eqs. (12).

The idler and signal branches discussed above [see in Figs. 2b 3 are the eigenvalues of Eq. (4) calculated with $\mathcal{P}_{0}^{2}=0.14$, a threshold value for the stimulated scattering into $k_{s}<0, k_{i}>2 k_{0}$. And indeed, Fig. $4 b$ shows that at $t<600$ ps the scattered noise is maximum at the predicted angles $k_{s}<0, k_{i}>2 k_{0}$. However, at $t \approx 600 \mathrm{ps}$, instead of the stimulated scattering into these modes, another instability develops. The trajectory of the system on the $\left[\left|\mathcal{P}\left(k_{0}, t\right)\right|^{2},\left|\mathcal{E}_{\text {ext }}(t)\right|^{2}\right]$ plane, see in Fig. $4 k$, shows that the instability at $t \approx 600 \mathrm{ps}$ is the jump of the $k_{0}$ mode between the lower and upper branches of the Sshaped curve.

In the empty cavity with quadratic dispersion, the upper S' branch is usually stable [16, 17]. In a MC with inflective LP dispersion, it can become unstable against parametric scattering. It can be seen from, e.g., Fig. 2 $c$, showing the signal and idler branches calculated on the upper part of S-curve at $\left|\mathcal{P}_{0}\right|^{2}=0.44$. Instead of damping, large gain is realized for polariton modes just with $k_{s} \gtrsim 0, k_{i} \lesssim 2 k_{0}$. As a result, a stimulated scattering into these modes develops (see in Fig. 4b). It is feed up by the increased absorption, see dashed line in Fig. $4 a$. But the pumped mode becomes unstable and eventually jumps back into the lower position (at $t \approx 700 \mathrm{ps}$ ). Now, because we already have a well developed scattered noise in the system, this lower position is more or less stable, although subject to noisy fluctuations because of the already developed scattered states and the competition between them and the pumped mode.

We would like to note that within our theoretical model the instability of the upper S' branch takes place in a narrower region of parameters than in the experiment. E.g., for the pump at $\vartheta_{0}=14^{\circ}$ and $12.5^{\circ}$ (as in the experiment in Fig. 16) it occurs for detuning $0.1<\Delta<$ $0.6 \mathrm{meV}$ and $0.15<\Delta<0.3 \mathrm{meV}$, respectively. However, the other scattering mechanisms, e.g., with phonons and free carriers, which are neglected in our approach, can increase substantially the region of the instability.

To conclude, we have found experimentally that above the MC parametric scattering threshold the signal and idler maxima do not shift along the renormalised LP branch but jump to $k_{s} \gtrsim 0, k_{i} \lesssim 2 k_{0}$ directions. We show that qualitatively this behavior can be explained via an interplay between the the bistability of the driven mode itself and the stimulated parametric scattering. The bistability, although well known in empty nonlinear cavities, was not accounted for in the existent theoretical models of the MC polaritons parametric scattering. It acquires a new feature in $\mathrm{MC}$ with polariton resonance: instability of the upper branch of the S-shaped dependence of pumped mode polarisation vs pump. Although the exciton-exciton scattering may be not the only mechanism causing the instability, this feature is important and has to be taken into account.

We thank L. V. Keldysh for stimulating discussions, and M. S. Skolnick for samples and discussions. This work was financially supported in part by the Russian Foundation for Basic Research, Russian Ministry of Science, and INTAS.

[1] P. G. Savvidis et al., Phys. Rev. Lett. 84, 1547 (2000). 
[2] R. Houdré et al., Phys. Rev. Lett. 85, 2793 (2000).

[3] C. Ciuti et al., Phys. Rev. B 62, R4825 (2000).

[4] A. I. Tartakovskii et al., Phys. Rev. B 62, R13298 (2000).

[5] R. M. Stevenson et al., Phys. Rev. Lett. 85, 3680 (2000).

[6] J. J. Baumberg et al., Phys. Rev. B 62, R16247 (2000).

[7] C. Ciuti et al., Phys. Rev. B 63, 041303 (2001).

[8] D. M. Whittaker, Phys. Rev. B 63, 193305 (2001).

[9] P. G. Savvidis et al., Phys. Rev. B 64, 075311 (2001).

[10] M. Saba et al., Nature 414, 731 (2001).
[11] S. Savasta et al., Phys. Rev. Lett. 90, 096403 (2003).

[12] A. Huynh et al., Phys. Rev. Lett. 90, 106401 (2003).

[13] V. D. Kulakovskii et al., Nanotechnology 12, 475 (2001).

[14] C. Piermarocchi et al., Phys. Rev. B 53, 15834 (1996).

[15] G. Malpuech et al., Phys. Rev. B 65, 153310 (1996).

[16] W. J. Firth and A. J. Scroggie, Phys. Rev. Lett. 76, 1623 (1996)

[17] R. Kuszelewicz et al., Phys. Rev. Lett. 84, 6006 (2000). 